\section{BRAZIULIAN JOURNAL \\ OF MEDICAL AND BIOLOGICAL RESEARCH}

www.bjournal.com.br
ISSN 1414-431X

Volume 45 (11) 995-1101 November 2012

BIOMIDICAL SCIENCES

AND

CLINICAL INVESTIGATION

Braz J Med Biol Res, November 2012, Volume 45(11) 995-1001

doi: $10.1590 / \mathrm{S} 0100-879 X 2012007500126$

RNAi-mediated knockdown of pituitary tumor-transforming gene-1 (PTTG1) suppresses the proliferation and invasive potential of PC3 human prostate cancer cells

S.Q. Huang, Q.J. Liao, X.W. Wang, D.Q. Xin, S.X. Chen, Q.J. Wu and G. Ye

The Brazilian Journal of Medical and Biological Research is partially financed by

\section{Q}

da Ciência e Tecnologia

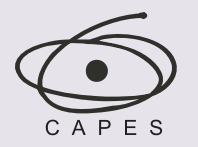

Ministério da Educação

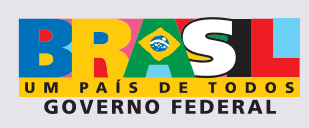

DTAPESP

Institutional Sponsors

๑ SHIMADZU UNICAMP

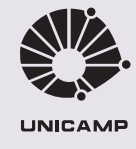

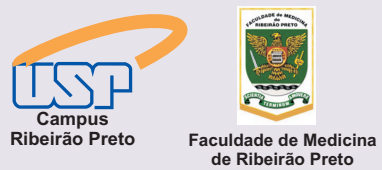

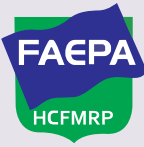




\title{
RNAi-mediated knockdown of pituitary tumor- transforming gene-1 (PTTG1) suppresses the proliferation and invasive potential of PC3 human prostate cancer cells
}

\author{
S.Q. Huang ${ }^{1,2}$, Q.J. Liao ${ }^{1}$, X.W. Wang ${ }^{1}$, D.Q. Xin ${ }^{2}$, S.X. Chen ${ }^{1}$, Q.J. Wu ${ }^{1}$ \\ and $G . Y e^{1}$ \\ ${ }^{1}$ Department of Urology and Center of Nephrology, Xinqiao Hospital, \\ Third Military Medical University, Chongqing, China \\ ${ }^{2}$ Institute of Urology, Peking University and Department of Urology, \\ First Hospital, Peking University, Beijing, China
}

\begin{abstract}
Pituitary tumor-transforming gene-1 (PTTG1) is a proto-oncogene that promotes tumorigenesis and metastasis in numerous cell types and is overexpressed in a variety of human tumors. We have demonstrated that PTTG1 expression was up-regulated in both human prostate cancer specimens and prostate cancer cell lines. For a more direct assessment of the function of PTTG1 in prostate tumorigenesis, RNAi-mediated knockdown was used to selectively decrease PTTG1 expression in PC3 human prostate tumor cells. After three weeks of selection, colonies stably transfected with PTTG1-targeted RNAi (the knockdown PC3 cell line) or empty vector (the control PC3 cell line) were selected and expanded to investigate the role of PTTG1 expression in PC3 cell growth and invasion. Cell proliferation rate was significantly slower (28\%) in the PTTG1 knockdown line after 6 days of growth as indicated by an MTT cell viability assay $(P<0.05)$. Similarly, a soft agar colony formation assay revealed significantly fewer (66.7\%) PTTG1 knockdown PC3 cell colonies than control colonies after three weeks of growth. In addition, PTTG1 knockdown resulted in cell cycle arrest at G1 as indicated by fluorescence-activated cell sorting. The PTTG1 knockdown PC3 cell line also exhibited significantly reduced migration through Matrigel in a transwell assay of invasive potential, and down-regulation of PTTG1 could lead to increased sensitivity of these prostate cancer cells to a commonly used anticancer drug, taxol. Thus, PTTG1 expression is crucial for PC3 cell proliferation and invasion, and could be a promising new target for prostate cancer therapy.
\end{abstract}

Key words: PTTG1; Prostate cancer; Cell proliferation; Transwell invasion assay; Anticancer drug resistance

\section{Introduction}

Prostate cancer is a common malignancy of the male urinary system (1). Prostate tumors have highly variable invasive potential, but there are few reliable biomarkers for distinguishing tumors that spontaneously enter a latent stage from those that continue to grow and metastasize. Androgens are important for the maintenance of prostate structure and function, and may also regulate prostate tumorigenesis (2-4). Indeed, many current treatments for inhibiting prostate tumor growth suppress testosterone signaling. However, this form of treatment shows reduced efficacy after several years, and the majority of cases eventually become androgen-independent (2). The identification of alternative pathways that regulate prostate tumor growth and metastasis could lead to the development of new prognostic markers and therapeutic targets.

Pituitary tumor-transforming gene-1 (PTTG1) is a recently identified oncogene (5) that is overexpressed in a broad range of cancerous tissues, including oral and esophageal squamous cell carcinomas $(6,7)$, pituitary adenomas (8-10), ovarian cancers (11), thyroid cancers (12), hepatocellular carcinomas (13), breast cancers (14), hematopoietic cancers (15), and gliomas (16). Furthermore,

Correspondence: X.W. Wang, Department of Urology and Center of Nephrology, Xinqiao Hospital, Third Military Medical University, Chongqing 400037, China. Fax: +86-023-6875-5683. E-mail: doctorwangxianwei@163.com

Received January 10, 2012. Accepted July 23, 2012. Available online August 10, 2012. Published October 5, 2012. 
high PTTG1 expression correlates with poor clinical outcome $(7,16)$, possibly because PTTG1 overexpression can lead to sustained overexpression of mitogens like c-Myc and FGF2 (17), inhibition of tumor suppressor and pro-apoptotic pathways (18), or reduced sensitivity to anti-neoplastic drugs $(14,19)$. In contrast to cancer cells, PTTG1 is only weakly expressed or is undetectable in most healthy adult human tissues (e.g., healthy colon and pituitary gland). Expression in healthy tissue is restricted to proliferating tissues like normal fetal liver, testis, and thymus $(9,15)$.

Analysis of PTTG1 expression in various cell lines, as well as ectopic overexpression and knockdown studies, strongly suggests that PTTG1 promotes malignant transformation. A variety of transformed cell lines overexpress PTTG1 $(18,20)$, and often exhibit altered expression of other mitogenic, apoptotic, and angiogenic genes (21). Alternatively, overexpression can lead to cell transformation and tumorigenesis without the concurrent activation of other oncogenes (8). Thymectomized nude mice injected subcutaneously with NIH3T3 fibroblasts overexpressing PTTG developed tumors within three weeks $(8,15)$. In addition to enhanced cell proliferation, PTTG1 overexpression reduced UV-induced apoptosis in vitro (20), while suppression of PTTG1 enhanced apoptosis $(18,20)$. Overexpression of PTTG1 is activated by the STAT5 signaling pathway (22), and PTTG1 overexpression is associated with reduced expression of the ubiquitous tumor suppressors p53 and p21 (17). Moreover, several cell senescence molecules, including Kruppel-like factor 6 (23), may act by suppressing PTTG1 expression. Moreno-Mateos et al. (24) recently demonstrated that PTTG1 is a multifunctional protein with distinct nuclear and cytoplasmic functions controlled by phosphorylation (24). Cytoplasmic PTTG1 controls the nucleation of microtubules, and PTTG1 knockdown impedes the development of cell polarity and cell migration in wound assays. Thus, PTTG1 overexpression may enhance the invasive potential of cancer cells by regulating cytoskeletal dynamics.

We have demonstrated that PTTG1 is overexpressed in the human prostate cancer cell lines LNCaP, PC3, and DU145, and is a novel androgen-responsive gene in rat prostate and LNCaP cells. We also found that up-regulated PTTG1 expression correlated with higher grade prostate cancer $(25,26)$. In the present investigation, we studied the effects of PTTG1 expression on cell growth, invasion, and cell cycle progression in the androgen-independent human prostate cell line PC3 using a specifically targeted RNAi.

\section{Material and Methods}

\section{Cell cultures}

Human PC3 prostate cancer cells were obtained from the American Type Culture Collection (USA) and grown in RPMI 1640 medium supplemented with 10\% (v/v) fetal bo- vine serum (Invitrogen, USA), glutamine ( $2 \mathrm{mM}$; Invitrogen), and penicillin plus streptomycin $(100 \mathrm{U} / \mathrm{mL}$ and $100 \mu \mathrm{g} / \mathrm{mL}$; Invitrogen) at $37^{\circ} \mathrm{C}$ in a $5 \% \mathrm{CO}_{2}$ atmosphere.

\section{Knockdown of PTTG1}

To obtain a stable PTTG1 knockdown cell line, according to Ref. 20, two single-strand oligonucleotides were synthesized: top-strand 5'-CACCGTCCTCTAGACTT TGAGAGTTTCAAGAGAACTCTCAAAGTCTAGAGG ATTTTTTGGAA-3' and lower-strand 5'-AAAATTCCAA AAAATCCTCTAGACTTTGAGAGTTCTCTTGAAACTCTC AAAGTCTAGAGGAC-3'. These 2 oligonucleotides were annealed to form duplexes. The duplex products were subcloned into the $p$ Silencer 2.0 vector and correct insertion and orientation were confirmed by sequencing. The PC3 cells were transfected with p Silencer 2.0-PTTG1 plasmid using Lipofectamine 2000 (Invitrogen) according to the protocols of the manufacturer. After $48 \mathrm{~h}$, the cell medium was changed to RPMI 1640 containing $2 \mu \mathrm{g} / \mathrm{mL}$ puromycin (Invitrogen) and cells were selected for 3 weeks. In the present study, the puromycin-resistant PTTG1 knockdown cells are named PTTG1RNAi cells. The stable PTTG1RNAi cells were maintained in RPMI 1640 medium supplemented with $0.5 \mu \mathrm{g} / \mathrm{mL}$ puromycin. A cell line stably expressing the empty p Silencer 2.0 vector was used as the control. Western blotting was performed to detect PTTG1 protein expression in these two cell lines.

\section{Western blotting}

Whole cell protein extracts were obtained by lysing exponentially growing cells in a RIPA buffer containing 50 $\mathrm{mM}$ Tris- $\mathrm{HCl}, \mathrm{pH} 7.4,150 \mathrm{mM} \mathrm{NaCl}, 10 \mathrm{mM}$ EGTA, 1\% NP-40, 0.1\% SDS, $1 \mathrm{mM} \mathrm{Na}_{3} \mathrm{VO}_{4}, 50 \mathrm{mM} \mathrm{NaF}, 2 \mu \mathrm{g} / \mathrm{mL}$ aprotinin, $2 \mu \mathrm{g} / \mathrm{mL}$ leupetin, and $1 \mathrm{mM}$ phenylmethylsulfonyl fluoride. Lysates were centrifuged at 16,000 $\mathrm{g}$ in an Eppendorf $5417 \mathrm{R}$ centrifuge at $4^{\circ} \mathrm{C}$ for $15 \mathrm{~min}$, and total protein concentrations in the supernatants were determined using a bicinchoninic acid protein assay (Pierce, USA). $\beta$-actin expression was used as an internal control to normalize protein content.

Proteins were separated by $15 \%$ SDS-polyacrylamide gel electrophoresis at $30 \mu \mathrm{g}$ per gel lane, and transferred onto nitrocellulose membranes (Whatman Inc., USA). Membranes were then blocked in Tris-buffered saline with $0.1 \%$ Tween 20 containing $5 \%$ nonfat milk and probed with a primary PTTG1 antibody (1:500; Santa Cruz, USA; sc-33099) at $4^{\circ} \mathrm{C}$ overnight. Immune complexes were detected using horseradish peroxidaseconjugated donkey anti-rabbit or anti-goat IgG (Amersham Biosciences, Baie d'Urfé, Canada) followed by chemiluminescence (Pierce) detection by exposure to $X$-ray film (Kodak, USA). $\beta$-actin expression was used as the internal control using anti- $\beta$-actin (Cell Signaling \#4967). Each Western blotting trial was repeated three times on different PC3 cultures. 


\section{Cell proliferation assay and cell cycle analysis}

Cell proliferation was assessed using the MTT-based CellTiter 96 Non-Radioactive Cell Proliferation Assay Kit (Promega, USA) according to manufacturer protocol. For analysis of the cell cycle, cells were washed with ice-cold PBS, detached with $0.25 \%$ Trypsin, and fixed with $70 \%$ ethanol overnight at $4^{\circ} \mathrm{C}$. Fixed cells were subsequently washed, treated with $5 \mu \mathrm{g} / \mathrm{mL}$ RNase A (Sigma, USA), and stained with $50 \mu \mathrm{g} / \mathrm{mL}$ propidium iodide (Sigma). Stained cells were assayed in a fluorescence-activated cell sorter (Becton Dickinson, USA) and the data were analyzed using the Cell FIT software. A minimum of 10,000 cells were analyzed in each sample.

\section{Soft agar colony formation assay}

A soft agar colony formation assay was performed as described (27). Briefly, 6-well plates were coated with a bottom layer of $1.5-\mathrm{mL}$ base agar consisting of $0.5 \%$ agar, 1X RPMI 1640 medium, and 10\% fetal bovine serum (Invitrogen), followed by a top layer of $1 \mathrm{~mL}$ $0.35 \%$ agar. In each well, $5 \times 10^{3}$ cells (either control or PTTG1RNAi cells) were plated over the top layer. Each assay was performed in triplicate wells for both cell lines. Plates were assessed for size and number of colonies under a Zeiss microscope after three weeks of incubation. All experiments were performed three times using different cultures.

\section{Transwell invasion assay}

The transwell invasion assay was performed according to a previous report (28). Briefly, $1 \times 10^{5}$ cells were seeded onto the upper surface of a transwell insert (Costar, USA) precoated with the extracellular matrix substitute Matrigel (BD Biosciences, USA) and these upper chamber cells were incubated in serum-free RPMI 1640 medium. The lower chamber contained RPMI 1640 medium supplemented with $10 \%$ fetal bovine serum (Invitrogen). After incubation for 24 $\mathrm{h}$, non-migratory cells on the upper surface were completely removed with a cotton swab. Cells on the lower surface of the insert were stained with $0.1 \%$ crystal violet for 20 min and cell number was counted under a microscope. Each cell line was plated in triplicate and each experiment was repeated three times.

\section{MTT assay estimate the sensitivity of PC3 cells to the hemotherapy drug taxol}

Cell proliferation was assessed using the MTT-based CellTiter 96 Non-Radioactive Cell Proliferation Assay Kit (Promega) according to the manufacturer protocol and previously described experimental procedures (29). Briefly, 3000 cells were seeded on 96-well plates and cultured for 24 $\mathrm{h}$ and four taxol concentrations $(5,10,20,40 \mathrm{ng} / \mathrm{mL})$ were added. Cell viability was examined $24 \mathrm{~h}$ after treatment. The results represent the ratio of the absorbance at 490 $\mathrm{nM}$ of the treated and untreated cells, at the indicated time points. Each data point is reported as the mean and standard deviation. The experiment was repeated six times.

\section{Statistical analysis}

Data are reported as means $\pm S D$. Mean differences were compared by ANOVA and the Student $t$-test. A P value of less than 0.05 was considered to be statistically significant.

\section{Results}

\section{PTTG1 knockdown inhibited PC3 cell proliferation}

To determine whether PTTG1 expression is related to the rate of $\mathrm{PC} 3$ cell proliferation, MTT cell viability assays were performed daily on control and PTTG1 knockdown cell lines for 7 days after seeding. We first determined by Western blotting that the cell line stably expressing a PTTG1targeted RNAi (PTTG1RNAi line) exhibited reduced PTTG1 expression compared to control cells (Figure 1A). The rate of cell proliferation was then estimated by measuring the conversion of MTT to formazan by viable cells after each day of growth in vitro (Figure 1B). The PTTG1RNAi line exhibited slower proliferation as indicated by the reduced formazan accumulation (measured by absorbance at 490 $\mathrm{nm}$ ) by day 6 in vitro (days 6 and $7, \mathrm{P}<0.05$ ).

\section{PTTG1 knockdown reduced PC3 cell colony formation}

A soft agar colony formation assay confirmed the reduced proliferative potential of the PTTG1RNAi cell line (Figure 2). Low-density cell suspensions were cultured for three weeks on soft agar substrate supplemented with growth medium and serum. Typical low-power microscopic fields of soft agar wells (Figure 2A) illustrate the reduction in PTTG1RNAi cell colony number compared to control colony number. On average, the PTTG1 knockdown PC3 cell line formed fewer colonies $(3.4 \pm 0.4$ colonies per low power field) than the control line stably transfected with the empty vector (Figure 2B; $\mathrm{P}<0.05$ ). Only colonies larger than $1 \mathrm{~mm}$ in diameter were counted.

\section{PTTG1 knockdown caused cell cycle arrest in G1}

We then compared the fractions of propidium iodidestained PTTG1RNAi and control PC3 cells in each phase of the cell cycle by fluorescence-activated cell sorting (Figure 3). A significantly greater fraction of PTTG1RNAi cells were in G1 (68.67\%) compared to control cells, while a lower percentage were in S + G2 (31.33\%) compared to control cells, suggesting that PTTG1 knockdown results in G1 cell cycle arrest.

\section{PTTG1 knockdown reduced the invasive capacity of PC3 cells}

To examine the effect of PTTG1 expression on the invasive capacity of $\mathrm{PC} 3$ cells, control and 
PTTG1RNAi cell lines were compared in a transwell invasion assay (Figure 4). Typical images of crystal violet-stained cells on the underside of a Matrigel-coated transwell membrane (Figure 4A) illustrate the reduced number of PTTG1RNAi cells that migrated across the Matrigel after 24 h. On average, significantly fewer PTTG1RNAi cells (26 \pm 7 cells/field) migrated through the Matrigel compared to control cells ( $47 \pm 6$ cells/ field, Figure 4B; $\mathrm{P}<0.05$ ).

\section{Down-regulation of PTTG1 leads to increased sensitivity to taxol}

To further investigate the possibility of using PTTG1 as a target to increase chemosensitivity in PC3 cells we performed the MTT assay to investigate whether down-regulation of PTTG1 had any effect on cell viability, in response to taxol compared to the vector control and parental PC3. As shown in Figure 5, downregulation of PTTG1 by RNAi was associated with decreased cell viability of PC3 cells in a dose-dependent manner in response to taxol treatment compared with the parental lines and vector controls. The cell viability of PTTG1 was lower than that of the controls after treatment with taxol. The difference was significant at higher drug concentrations $(20,40 \mathrm{ng} / \mathrm{mL}$; $\mathrm{P}<$ $0.05)$. The results indicate that down-regulation of PTTG1 in PC3 cells leads to decreased cell viability in response to taxol.

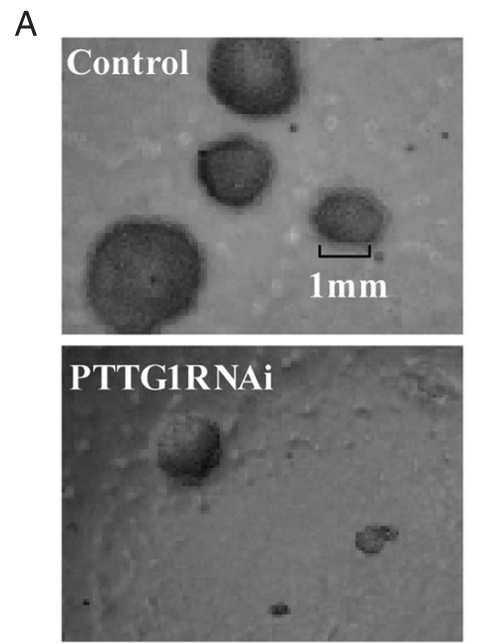

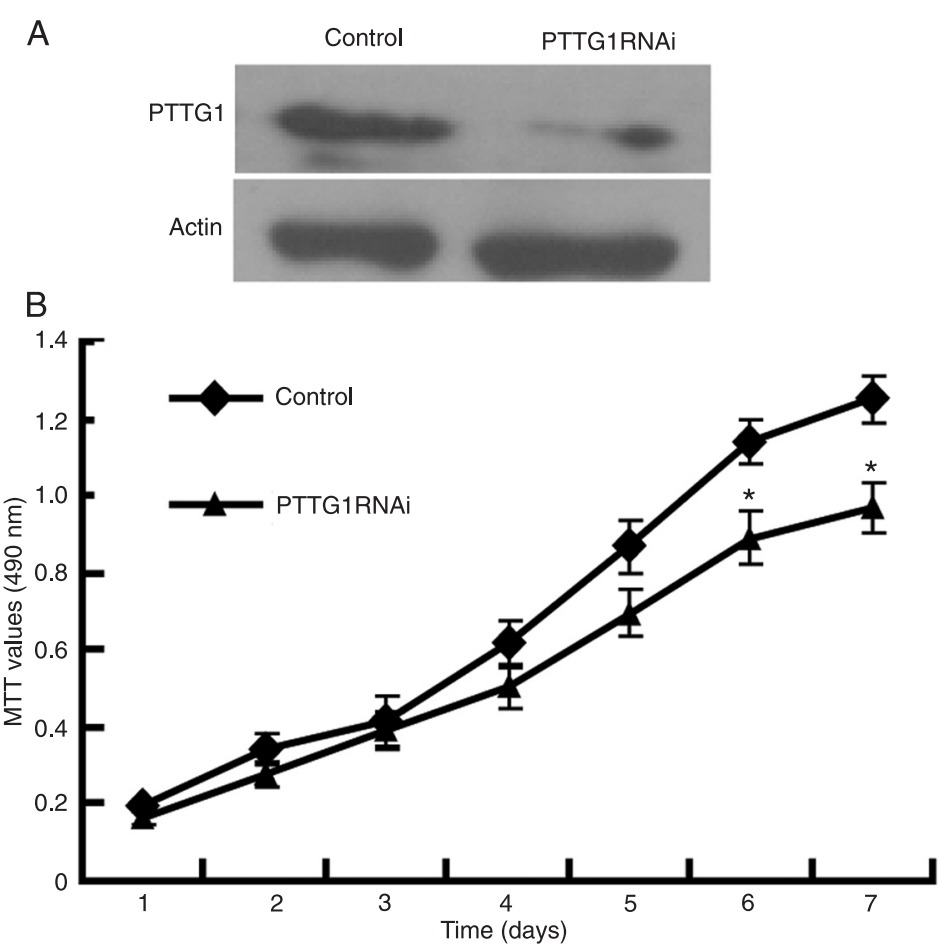

Figure 1. Pituitary tumor-transforming gene-1 (PTTG1) knockdown reduced PC3 proliferation. A, Reduced expression of PTTG1 protein in PC3 cells stably transfected with an RNAi targeting PTTG1 (PTTG1RNAi cells) compared to control cells expressing the empty vector. A representative Western blot of 3 samples is shown. $B$, An MTT assay was used to evaluate the proliferation rates of the PTTG1RNAi and control cell lines. The number of viable cells, an index of proliferation, was significantly reduced in PTTG1RNAi cultures compared to control on days 6 and 7 of culture. ${ }^{*} \mathrm{P}<0.05$ compared to control (Student $t$-test).

Figure 2. Pituitary tumor-transforming gene-1 (PTTG1) knockdown reduced PC3 cell colony formation. A, Colonies of PTTG1RNAi and control PC3 cells larger than $1 \mathrm{~mm}$ in diameter were counted. The light micrographs show colonies formed after 3 weeks of growth on soft agar. $B$, The number of $\mathrm{PC} 3$ colonies was significantly reduced by PTTG1 knockdown ( ${ }^{*} \mathrm{P}<0.05$, Student $t$-test) compared to control. The bar graph shows the mean number of colonies in 10 low-power fields for each cell line. 

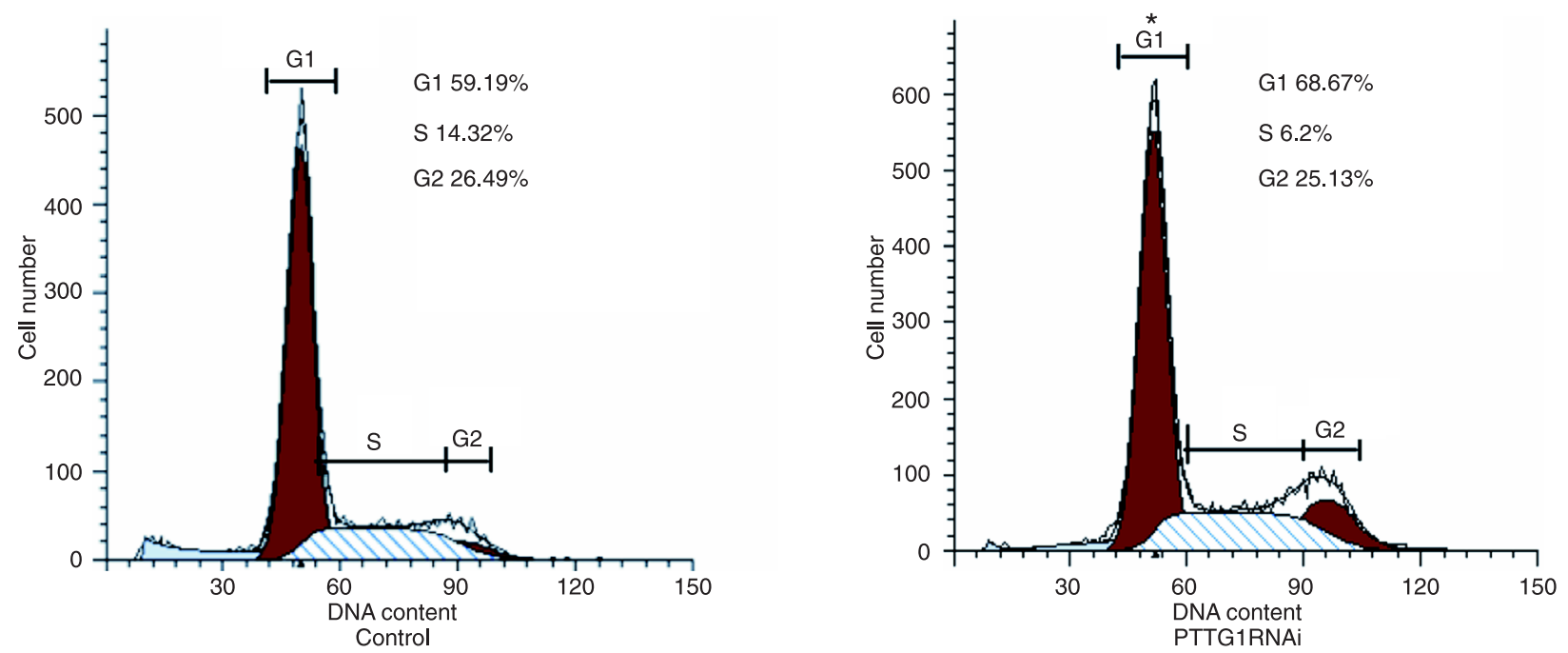

Figure 3. Pituitary tumor-transforming gene-1 (PTTG1) knockdown caused G1 cell cycle arrest. After staining with propidium iodide, cells were subjected to fluorescence-activated cell sorting analysis. Data are reported as means $\pm \mathrm{SD}$. * $<<0.05$ compared to control (Student $t$-test).

A
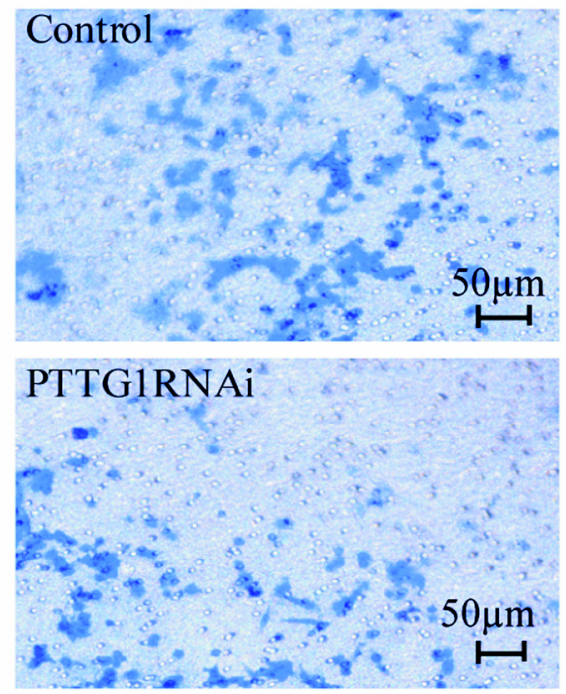

B

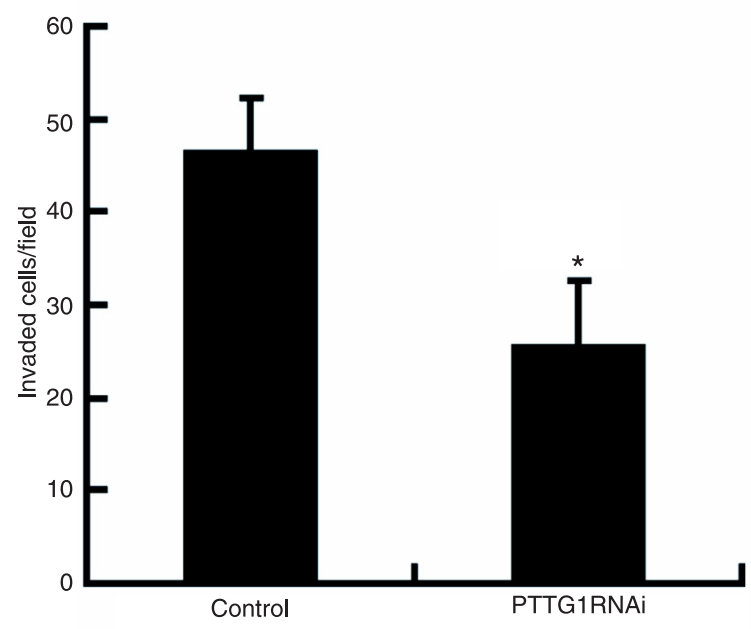

Figure 4. Pituitary tumor-transforming gene-1 (PTTG1) knockdown reduced invasive capacity as revealed by a transwell invasion assay. A, A significantly greater number of cells (stained blue) were observed on the underside of the Matrigel in transwell assays of control cultures than in transwell assays of PTTG1RNAi cultures. B, Quantification of the numbers of PTTG1RNAi and control PC3 cells migrating through the Matrigel. ${ }^{*} \mathrm{P}<0.05$ compared to control (Student $t$-test).

\section{Discussion}

Studies on the genesis and progression of prostate cancers have mainly focused on the activation and expression of oncogenes. These studies have identified a myriad of such genes, including hereditary prostate cancer-1 on chromosome 1q (30), MAX-interacting protein-1 on chro- mosome 10q, and the KAl1 prostate cancer antimetastasis gene on chromosome $11 \mathrm{p}(31,32)$. In a previous study, we demonstrated that PTTG1 promoted the proliferation of the prostate cell line LNCaP. In the present study, we found that PTTG1 is required for PC3 prostate cell growth and invasion. In light of our results showing that PTTG1 is overexpressed in human prostate cancer tissue (26), the 
present results strongly suggest that PTTG1 is a critical mediator of prostate tumor growth and invasion.

PTTG1 is highly expressed in a variety of other human tumor tissues (10-12). In addition, PTTG1 can regulate the expression of many genes involved in proliferation and cell cycle control. For example, PTTG1 can activate the oncogene c-myc to facilitate the formation and progression of tumors (33). Furthermore, PTTG1 can facilitate the expression of $\beta-F G F$, a strong angiogenic factor critical for tumor metastasis (21). In our study, PTTG1 knockdown greatly reduced the proliferation rate and colony formation of PC3 cells (Figures 1 and 2). Aside from oncogene activation, PTTG1 participates in cell cycle regulation and cell senescence by restraining the expression of the ubiquitous tumor suppressors p53 and p21 (34). Our results showed that PTTG1 knockdown resulted in cell cycle arrest at G1 (Figure 3) and failure to reach the G2/M transition, thus explaining the inhibition of PC3 cell growth. Future studies are required to determine whether PTTG1 knockdown leads to reduced expression of proto-oncogenes or to increased expression of tumor suppressors like p53, or both.

Malik and Kakar (35) found that HEK293 cells overexpressing PTTG1 exhibited enhanced expression and secretion of matrix metalloproteinase 2 (MMP-2), an extracellular protease involved in tissue remodeling, when injected into subcutaneous tumors of nude mice. Thus, in addition to effects on cell cycle progression, PTTG1 may facilitate the migration and metastasis of tumor cells by up-regulating the expression and secretion of MMP-2 (35). Consistent with previous reports showing that PTTG1 can increase MMP-2 expression and activity, we found that knockdown of PTTG1 expression in PC3 cells suppressed the invasive potential as evidence by a transwell assay (Figure 4). Thus, PTTG1 underexpression appears to inhibit tumor cell proliferation and migration. Again, further studies are required to elucidate the molecular mechanisms by which PTTG1 knockdown inhibits cell invasion.

The advanced stage of malignancy is always an obstruction in the treatment of prostate cancer. The major stumbling block is the tendency of prostate cancer to transform to androgen reflexive after an initial period of remission after androgen ablation therapy. In addition, chemotherapy has not shown good results in prostate cancer, probably due to the relatively slow growing nature of the tumor and the elderly age of the patients. Also, some investigations have considered anticancer drug resistance in prostate cancer to be related to the expression of certain genes. In the present

\section{References}

1. Hsing AW, Tsao L, Devesa SS. International trends and patterns of prostate cancer incidence and mortality. Int $J$ Cancer 2000; 85: 60-67.

2. Brown TR, Lubahn DB, Wilson EM, Joseph DR, French FS,

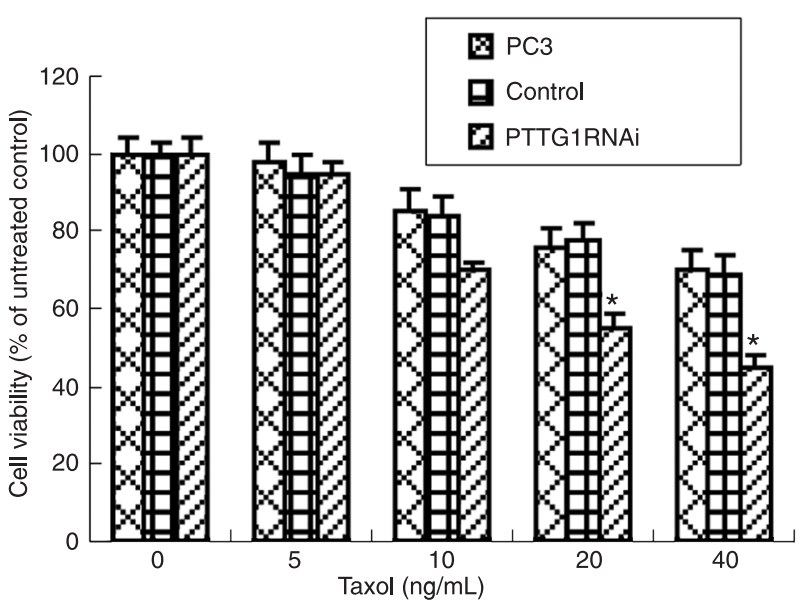

Figure 5. Viability of pituitary tumor-transforming gene-1 RNAi (PTTG1RNAi), vector controls and parental PC3 after exposure to taxol as determined by the MTT assay. Cells were seeded on 96 -well plates and drugs were added $24 \mathrm{~h}$ later. Cell viability was examined $24 \mathrm{~h}$ after treatment. Results are reported as the absorbance ratio of treated and untreated cells. Note that cell viability of PTTG1RNAi is lower than that of the vector controls and parental PC3. ${ }^{*} \mathrm{P}<0.05$ compared to the vector control and parental PC3 (ANOVA).

study, using the MTT assay, we have demonstrated that inactivation of PTTG1 in androgen-independent prostate cancer cells is able to induce sensitivity to taxol, a commonly used anticancer drug, (Figure 5) and PTTG1 may be a novel therapeutic target for prostate cancer. A more in-depth study will confirm this and determine that the mechanism of PTTG1 down-regulation leads to increased sensitivity to anticancer drugs.

In conclusion, our observations suggest that PTTG1 knockdown can significantly reduce proliferation rate, disrupt cell cycle progression, inhibit cell invasion, and increased sensitivity to anticancer drugs. In light of our previous study (26) demonstrating PTTG1 overexpression in prostate tumors but little or no expression in healthy tissue, these results suggest that PTTG1 may be a valuable prognostic marker and a novel therapeutic target in prostate cancer.

\section{Acknowledgments}

Research supported by the National Natural Science Foundation of China (\#81172443).
Migeon CJ. Deletion of the steroid-binding domain of the human androgen receptor gene in one family with complete androgen insensitivity syndrome: evidence for further genetic heterogeneity in this syndrome. Proc Natl Acad Sci $U$ 
S A 1988; 85: 8151-8155.

3. Cinar B, Sikes RA. Control of steroid hormone receptor action. Cancer Res 1999; 1: 100-103.

4. Yong EL, Lim J, Qi W, Ong V, Mifsud A. Molecular basis of androgen receptor diseases. Ann Med 2000; 32: 15-22.

5. Pei L, Melmed S. Isolation and characterization of a pituitary tumor-transforming gene (PTTG). Mol Endocrinol 1997; 11: 433-441.

6. Liao LJ, Hsu YH, Yu CH, Chiang CP, Jhan JR, Chang LC, et al. Association of pituitary tumor transforming gene expression with early oral tumorigenesis and malignant progression of precancerous lesions. Head Neck 2011; 33: 719-726.

7. Ito T, Shimada Y, Kan T, David S, Cheng Y, Mori Y, et al. Pituitary tumor-transforming 1 increases cell motility and promotes lymph node metastasis in esophageal squamous cell carcinoma. Cancer Res 2008; 68: 3214-3224.

8. McCabe CJ, Gittoes NJ. PTTG - a new pituitary tumour transforming gene. J Endocrinol 1999; 162: 163-166.

9. Zhang X, Horwitz GA, Prezant TR, Valentini A, Nakashima $M$, Bronstein MD, et al. Structure, expression, and function of human pituitary tumor-transforming gene (PTTG). Mol Endocrinol 1999; 13: 156-166.

10. Zhang X, Horwitz GA, Heaney AP, Nakashima M, Prezant TR, Bronstein MD, et al. Pituitary tumor transforming gene (PTTG) expression in pituitary adenomas. J Clin Endocrinol Metab 1999; 84: 761-767.

11. Shah PP, Kakar SS. Pituitary tumor transforming gene induces epithelial to mesenchymal transition by regulation of Twist, Snail, Slug, and E-cadherin. Cancer Lett 2011; 311: 66-76.

12. Smith VE, Franklyn JA, McCabe CJ. Pituitary tumor-transforming gene and its binding factor in endocrine cancer. Expert Rev Mol Med 2010; 12: e38.

13. Fujii T, Nomoto S, Koshikawa K, Yatabe Y, Teshigawara O, Mori T, et al. Overexpression of pituitary tumor transforming gene 1 in $\mathrm{HCC}$ is associated with angiogenesis and poor prognosis. Hepatology 2006; 43: 1267-1275.

14. Ghayad SE, Vendrell JA, Bieche I, Spyratos F, Dumontet C, Treilleux I, et al. Identification of TACC1, NOV, and PTTG1 as new candidate genes associated with endocrine therapy resistance in breast cancer. J Mol Endocrinol 2009; 42: 87103.

15. Dominguez A, Ramos-Morales F, Romero F, Rios RM, Dreyfus F, Tortolero M, et al. hpttg, a human homologue of rat pttg, is overexpressed in hematopoietic neoplasms. Evidence for a transcriptional activation function of hPTTG. Oncogene 1998; 17: 2187-2193.

16. Genkai N, Homma J, Sano M, Tanaka R, Yamanaka R. Increased expression of pituitary tumor-transforming gene (PTTG)-1 is correlated with poor prognosis in glioma patients. Oncol Rep 2006; 15: 1569-1574.

17. Tong Y, Eigler T. Transcriptional targets for pituitary tumortransforming gene-1. J Mol Endocrinol 2009; 43: 179-185.

18. Cho-Rok J, Yoo J, Jang YJ, Kim S, Chu IS, Yeom YI, et al. Adenovirus-mediated transfer of siRNA against PTTG1 inhibits liver cancer cell growth in vitro and in vivo. Hepatology 2006; 43: 1042-1052.

19. Tong Y, Zhao W, Zhou C, Wawrowsky K, Melmed S. PTTG1 attenuates drug-induced cellular senescence. PLoS One 2011; 6: e23754.
20. Lai Y, Xin D, Bai J, Mao Z, Na Y. The important anti-apoptotic role and its regulation mechanism of PTTG1 in UV-induced apoptosis. J Biochem Mol Biol 2007; 40: 966-972.

21. Hamid T, Malik MT, Kakar SS. Ectopic expression of PTTG1/ securin promotes tumorigenesis in human embryonic kidney cells. Mol Cancer 2005; 4: 3.

22. Shen XL, Wei W, Xu HL, Zhang MX, Qin XQ, Shi WZ, et al. JAK2V617F/STAT5 signaling pathway promotes cell proliferation through activation of Pituitary Tumor Transforming Gene 1 expression. Biochem Biophys Res Commun 2010; 398: 707-712.

23. Lee UE, Ghiassi-Nejad Z, Paris AJ, Yea S, Narla G, Walsh $\mathrm{M}$, et al. Tumor suppressor activity of KLF6 mediated by downregulation of the PTTG1 oncogene. FEBS Lett 2010; 584: 1006-1010.

24. Moreno-Mateos MA, Espina AG, Torres B, del Estal MM, Romero-Franco A, Rios RM, et al. PTTG1/securin modulates microtubule nucleation and cell migration. Mol Biol Cell 2011; 22: 4302-4311.

25. Xin $D Q$, Zhu $X H$, Lai $Y Q$, You $R$, Na $Y Q$, Guo $Y L$, et al. [Regulation of expression of pituitary tumor transforming gene 1 (PTTG1) by androgen in prostate cancer]. Beijing Da Xue Xue Bao 2005; 37: 638-640.

26. Zhu X, Mao Z, Na Y, Guo Y, Wang X, Xin D. Significance of pituitary tumor transforming gene 1 (PTTG1) in prostate cancer. Anticancer Res 2006; 26: 1253-1259.

27. Ivanova AV, Goparaju CM, Ivanov SV, Nonaka D, Cruz C, Beck A, et al. Protumorigenic role of HAPLN1 and its $\lg \mathrm{V}$ domain in malignant pleural mesothelioma. Clin Cancer Res 2009; 15: 2602-2611.

28. Wen DS, Zhu XL, Guan SM, Wu YM, Yu LL, Wu JZ. Silencing of CXCR4 inhibits the proliferation, adhesion, chemotaxis and invasion of salivary gland mucoepidermoid carcinoma Mc3 cells in vitro. Oral Oncol 2008; 44: 545-554.

29. Zhang $X$, Ling MT, Wang X, Wong YC. Inactivation of Id-1 in prostate cancer cells: A potential therapeutic target in inducing chemosensitization to taxol through activation of JNK pathway. Int J Cancer 2006; 118: 2072-2081.

30. Scheuner MT, McNeel TS, Freedman AN. Population prevalence of familial cancer and common hereditary cancer syndromes. The 2005 California Health Interview Survey. Genet Med 2010; 12: 726-735.

31. Eagle LR, Yin X, Brothman AR, Williams BJ, Atkin NB, Prochownik EV. Mutation of the MXI1 gene in prostate cancer. Nat Genet 1995; 9: 249-255.

32. Park JJ, Jin YB, Lee YJ, Lee JS, Lee YS, Ko YG, Lee M. KAI1 suppresses HIF-1 $1 \alpha$ and VEGF expression by blocking CDCP1-enhanced Src activation in prostate cancer. BMC Cancer 2012; 12: 81.

33. Pei L. Identification of c-myc as a down-stream target for pituitary tumor-transforming gene. J Biol Chem 2001; 276: 8484-8491.

34. Chesnokova V, Zonis S, Rubinek T, Yu R, Ben-Shlomo A, Kovacs K, et al. Senescence mediates pituitary hypoplasia and restrains pituitary tumor growth. Cancer Res 2007; 67: 10564-10572.

35. Malik MT, Kakar SS. Regulation of angiogenesis and invasion by human pituitary tumor transforming gene (PTTG) through increased expression and secretion of matrix metalloproteinase-2 (MMP-2). Mol Cancer 2006; 5: 61. 